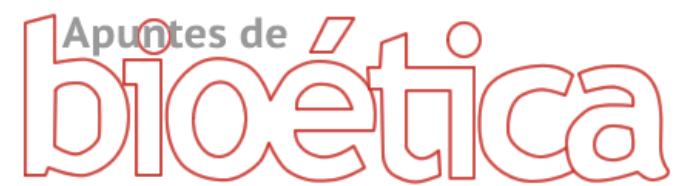

https://doi.org/10.35383/apuntes.v4i1.584

e-ISSN: $2663-4910$

Universidad Católica Santo Toribio de Mogrovejo

\title{
Perspectivas de profesionales peruanos sobre las declaraciones de voluntad anticipada
}

\section{Perspectives of Peruvian professionals on declarations of advance directives}

\author{
Yordanis Enriquez-Canto*, 1, a \\ yenriquez@ucss.edu.pe \\ https://orcid.org/0000-0003-0449-5015
}

Katia Denisse Chaparro-Gamarra ${ }^{1, b}$

kchaparro@ucss.edu.pe

https://orcid.org/0000-0001-6328-8409

\author{
* Autor corresponsal \\ ${ }^{1}$ Universidad Católica Sedes Sapientiae, Lima, Perú. \\ a Doctor en Bioética \\ ${ }^{\mathrm{b}}$ Maestra en Bioética y Bioderecho
}

Fechas importantes

Recibido: 2021-03-04

Aceptado: 2021-05-10

Publicado online: 2021-07-26

\section{Resumen}

Con el objetivo examinar las opiniones y perspectivas de médicos, abogados y notarios peruanos sobre las directivas anticipadas, a partir de la dación de la Ley 29633 , se realizó un estudio cualitativo. Se desarrollaron entrevistas semiestructuradas a 15 profesionales siguiendo un enfoque abierto con posterior análisis temático según Braun y Clarke. La mayoría de entrevistados eran varones. Las edades oscilaron entre 35 y 60 años, con experiencia laboral de 10 a 30 años. Se identificaron tres temas principales: respeto de la autonomía de la persona a través de una declaración de voluntad anticipada ejecutada por un familiar cercano; alcances de la manifestación de voluntad anticipada formalizada en instrumento público; respeto a la voluntad propia y a la voluntad ajena, con nueve subtemas. La comprensión de la perspectiva de los médicos y abogados constituye un respaldo a la discusión sobre las directivas anticipadas colaborando a cerrar las brechas legal y ética.

Palabras clave: Derechos civiles; Derecho de autodeterminación; Capacidad; Eutanasia.

\section{Abstract}

To examine the opinions and perspectives of Peruvian doctors, lawyers, and notaries on advance directives, based on the Law 29633, a qualitative study was carried out. Semi-structured interviews 
were conducted with 15 professionals following an open approach with subsequent thematic analysis according to Braun and Clarke. Most respondents were male. The ages ranged from 35 to 60 years, with work experience of 10 to 30 years. Three main themes were identified: respect for the person's autonomy through a declaration of advance directive will be executed by a close relative; the scope of the manifestation of the advance directive be formalized in a public instrument; respect for one's own will and the will of others, with nine sub-themes. Understanding the perspective of doctors and lawyers is a support for the discussion of advance directives by helping to close the legal and ethical gaps.

Keywords: Civil and political rights; Self-determination; Ability; Euthanasia.

\section{Introducción}

Las declaraciones de voluntad anticipada (DVA) son un tema multidimensional con un creciente historial en Bioética. Estas han sido definidas como un proceso que apoya a los adultos de cualquier edad o condición de salud a comprender, a partir de sus valores personales, objetivos de vida y preferencias respecto a la futura atención médica (Rietjens et al., 2017; Sudore et al., 2016). No obstante, en algunos contextos se admitan estas instrucciones de tratamiento su implementación es globalmente heterogénea (Nicholas et al., 2011). De hecho, en bioética y desde el punto de vista legal, representan un tema no exento de controversias. Al punto de que las regulaciones sobre el tema desvelan una doble necesidad por momentos contrapuesta. Por una parte, la obligación de ofrecer a los pacientes garantías a sus derechos frente al avance progresivo de la tecnología médica, por la otra la custodia de la pluralidad dentro de cada nación (Brighton y Bristowe, 2016; Brinkman et al., 2014).

Añadido a lo anterior, otros temas en el horizonte de la atención al final de la existencia añaden complejidad a las DVA, como son: las órdenes de no resucitar y la tan discutida y controversial eutanasia o de suicidio asistido. En este contexto permanece un vacío legislativo importante, aunque la ley pueda abordar algunos de estos aspectos en su totalidad o sólo parcialmente (Indabas et al., 2016; Indabas et al., 2019). Hasta el momento, y a nivel mundial, la reglamentación específica sobre directivas anticipadas está evolucionando. En este sentido, en Europa, los Países Bajos lideran con la promulgación de una ley sobre directivas anticipadas en 1994. En el 2017, Suecia e Italia también promulgaron leyes sobre el argumento. Mientras que, en América latina, también se evidencian avances legislativos consolidados (Monteiro et al., 2019).

A pesar de las diferencias locales, un elemento que las congrega es la creciente atención, debido a la gradual necesidad de medidas de signo positivo para proporcionar cuidados paliativos y tratamientos adecuados al final de la vida (Botti y Vaccari, 2019). Paralelamente 
a este incremento surgen preguntas a nivel ético y legal. En esta línea, se verifica un vacío reglamentario que ayude al personal sanitario a tomar decisiones en las situaciones donde los deseos del paciente no son del todo claros (Heyland et al., 2017; Tulsky et al., 2017). De manera similar, allí donde la interpretación de quienes deben tomar decisiones entra en conflicto respecto a las declaraciones anticipadas (Heyland et al., 2017; Tulsky et al., 2017). Añadido a lo anterior, en la atención sanitaria se reportan barreras a la clara implementación de las declaraciones anticipadas. Entre ellas se encuentra el nivel de conocimiento y la escasa familiaridad del personal médico con las legislaciones relativas a las DVA (Nehra y Gupta, 2019), el acceso por parte de los ciudadanos a las directivas anticipadas (Haesen y Shaw, 2018), la comprensión y la percepción de utilidad respecto a las mismas (Brinkman et al., 2014). No obstante, los elementos conflictuales descritos se ha señalado paralelamente que las DVA contribuyen a la calidad de vida de los pacientes cercanos al final de la existencia (Brinkman et al., 2014; Kernick et al., 2018), reduciendo los costos de la atención sanitaria (Nguyen et al., 2017). Estos datos llevan, de consecuencia, a que los sistemas nacionales de salud coloquen su atención en una planificación previa de los tratamientos de naturaleza médica.

En el Perú, igualmente, a nivel legislativo existen deficiencias respecto a la autodeterminación terapéutica. El vacío legal genera un ambiente de falta de protección frente al principio bioético de autonomía y, consecuentemente, el respeto a la dignidad de la persona. Dentro del marco legal peruano se contemplan dos declaraciones de voluntad anticipada: La donación de órganos y tejidos regulada mediante Ley 28189 (2004) -que actualmente ha concluido por ser una declaración en el Registro Nacional de Identificación y Estado Civil- y la Ley que fortalece la tutela del incapaz o adulto mayor (Ley 29633, 2010).

La Ley 29633 a través de la modificación de diversos artículos del Código Civil planteó el fortalecimiento de la tutela del adulto mayor o incapaz mediante el nombramiento de su curador en sede notarial. En la actualidad al "curador" también se le denomina "apoyo", esto porque en septiembre de 2018, el Decreto Legislativo 1384 -Decreto legislativo que reconoce y regula la capacidad jurídica de las personas con discapacidad en igualdad de condiciones(Decreto legislativo 1384, 2018) ha llegado a complementar de cierta manera la norma referida en primer término, haciendo una distinción importante, en primer término, pero aún incompleta respecto a las declaraciones de voluntad anticipada y a su tratamiento legal en el Perú.

No obstante, aunque se puede considerar esta última como un avance en materia de DVA, es mínima la evolución y la protección del derecho a la autodeterminación terapéutica 
respecto a otros países (Torres, 2015). Lo anterior es válido si se tiene en cuenta que la Ley 29633 excluye los peruanos en la mayoría de edad y menores de 65 años. Este es un período en el cual las personas generalmente tienen plena capacidad, lo que serviría como garantía para un correcto manejo de su autodeterminación terapéutica (Dabove, 2018). Esta diferenciación ha quedado en parte corregida por la dación del Decreto Legislativo 1384 Decreto legislativo que reconoce y regula la capacidad jurídica de las personas con discapacidad en igualdad de condiciones- (Decreto legislativo 1384, 2018). Sin embargo, el hecho de legislar únicamente para un determinado sector de la población -así se trate de población vulnerable-, pudiera perfilarse como una negación al respeto a la dignidad de la persona humana, en igualdad de condiciones.

El vacío peruano respecto a las DVA se presenta, por ejemplo, después de que una persona ha ingresado de manera voluntaria a un establecimiento de salud para seguir un procedimiento o algún tratamiento, pero, como consecuencia de estos, pierde la capacidad para tomar decisiones, permaneciendo en el establecimiento y requiriendo tratamiento especializado. El personal de salud tiene la obligación de informar tanto al paciente (en caso de poder tomar decisiones con ciertas limitaciones) como a los familiares (Varsi-Rospigliosi, 2001). Serán ellos quienes finalmente autorizarán la realización de tratamientos, terapias o intervenciones sobre el paciente (Varsi-Rospigliosi, 2001). Sin embargo, las decisiones que se toman pudieran estar en contradicción con sus valores personales, objetivos de vida y preferencias respecto a la futura atención sanitaria (Brighton y Bristowe, 2016; Tulski et al., 2017).

Si nos enfocamos directamente en la razón de ser de las DVA, rescatamos que el respeto a la dignidad de la persona se encuentra en la base y centro de este. Si entendemos la dignidad como "el derecho irrefragable a un determinado modo de existir" (García Toma y García Yzaguirre, 2018), podemos afirmar que cualquier manifestación destinada a hacer valer una decisión tomada de manera anticipada y debidamente informada es, en definitiva, una manera de preservar la dignidad de la persona.

Hay que tener presente, además, que la aplicación de las DVA únicamente si la persona ha perdido capacidad para poder manifestar su voluntad y hacer ejercicio de sus derechos como la autodeterminación, entendida como la "decisión individual o colectiva vinculada con el proyecto de vida existencial o coexistencial" (García Toma y García Yzaguirre, 2018), puede tener múltiples enfoques, siendo el que nos interesa en el presente estudio, el enfoque terapéutico. 
Así, la autodeterminación terapéutica, consiste en la decisión libre e informada de aceptar o declinar el empleo de un determinado acto médico, sujeto esto de manera indesligable al consentimiento informado, herramienta que sirve de protección tanto al personal de salud como a la persona que en determinada época se convierte en paciente.

Hasta el momento, en Perú no se encuentra disponible una base de datos nacional de directivas anticipadas. De consecuencia no es posible estimar, por ejemplo, cuántos adultos mayores y personas con discapacidad ya han decidido sobre el nombramiento de su apoyo en materia de tratamiento. Además, en lo particular, se sabe poco sobre las opiniones de los médicos, abogados y notarios sobre esta ley en lo referido a su aplicación. A fin de profundizar en la temática de las exigencias en la autodeterminación terapéutica y considerando la situación problemática planteada se propuso como objetivo examinar cualitativamente las opiniones y perspectivas de médicos, abogados, notarios, y registradores sobre la tutela del adulto mayor o incapaz a través del nombramiento de su propio curador en sede notarial (Ley 29633) en Perú.

\section{Material y métodos}

\section{Diseño:}

Se realizó un estudio cualitativo (Hernández Sampieri et al., 2004; Páramo Bernal, 2013) a fin de comprender las opiniones y perspectivas de los entrevistados respecto al tema. La recolección de los datos se llevó a cabo entre los meses de julio y noviembre del año 2017.

\section{Participantes:}

Los participantes fueron seleccionados mediante un muestreo no probabilístico, por criterio lógico (Hernández Sampieri et al., 2004; Páramo Bernal, 2013). Se entrevistó un total de 15 profesionales entre médicos (4), abogados (5), notarios (4) y registradores (2). Como criterio de elegibilidad se empleó la interacción con la norma desde cada una de sus profesiones.

\section{Técnicas de recolección de datos e instrumentos:}

Previo a la realización del estudio se identificó un conjunto claro de temas. Para el desarrollo del estudio se empleó la entrevista cara a cara (Hernández Sampieri et al., 2004), semiestructurada de preguntas mediante un enfoque abierto y no directivo. Se realizaron entrevistas en profundidad con la ayuda de un dispositivo de grabación de voz. Las entrevistas duraron en media entre treinta (30) a cuarenta y cinco (45) minutos. Añadido a lo anterior, se documentó toda la comunicación no verbal. Igualmente, se instó a los 
entrevistados a profundizar los argumentos que les resultaran relevantes y no necesariamente presentes en la guía de la entrevista.

El guion de la entrevista semiestructurada contó con un total de nueve preguntas abiertas y diferenciadas para cada grupo profesional a fin de obtener una visión profunda de las opiniones y experiencias de los entrevistados. Ejemplos de estas preguntas fueron: ¿Considera que el otorgante de la declaración de nombramiento de curador debería también declarar sobre aspectos referidos a su autodeterminación terapéutica? y Si fuera(n) su(s) padre(s) o su esposo (a) o su(s) hijo(s) ¿Ud. respetaría la declaración de voluntad anticipada otorgadas por él o ella y ejecutadas por su curador?

\section{Análisis de la información:}

Bajo el consentimiento de los participantes se grabaron los audios de las entrevistas. Posteriormente, fueron analizadas en detalle las transcripciones. Se empleó el análisis temático descrito por Braun y Clarke (2015). Fue desarrollado paulatinamente el marco de codificación empleando la lectura y relectura de las transcripciones. Finalmente, se estableció un conjunto de temas a partir de la información recolectada. Finalmente, el análisis de la información consideró la codificación sistemática e inductiva teniendo en cuenta la recopilación de los datos relevantes para el objetivo del estudio y la posterior codificación en temas (Vaismoradi et al. 2016).

\section{Aspectos éticos:}

El estudio contó con la aprobación del Comité de Ética de la Universidad Católica Sedes Sapientiae. Los participantes dieron su consentimiento verbal para la participación y se garantizó la confidencialidad de la información recolectada, así como el anonimato de los profesionales entrevistados. Del mismo modo, no se emplearon los nominativos de las personas involucradas y se omitieron nombres de instituciones que emergieron de las entrevistas.

\section{Resultados}

\section{Características de los entrevistados:}

De los 15 profesionales entrevistados 14 eran varones. Considerando la totalidad del grupo investigado sus edades oscilaron entre los 35 y los 60 años. Asimismo, la experiencia en el ejercicio laboral varió entre los 10 y los 30 años. Los médicos participantes son referentes peruanos en temas bioéticos, con estudios en bioética, y además especialistas en salud 
pública. El 50\% de ellos ejercía la docencia universitaria. Con relación a los abogados, cabe señalar que el ejercicio de su profesión lo desempeñaban en la magistratura. Entre ellos se encuentran, a su vez, especialistas en Derechos Humanos y en su totalidad ejercían la docencia universitaria.

Por su parte, entre los notarios se eligieron representantes de diferentes provincias peruanas (Barranca, Huaura, Huaral y Lima). Además del ejercicio de su función notarial, la mayoría de ellos ejercía la docencia universitaria. Para los registradores, se contó con la participación de un Jefe de una Oficina Registral de Lima, así como un abogado certificador de la misma Zona Registral.

\section{Opiniones y perspectivas de los entrevistados:}

Habiéndose analizado puntualmente las respuestas obtenidas por los entrevistados, se categorizaron las respuestas, identificándose tres temas principales: respeto de la autonomía de la persona a través de una declaración de voluntad anticipada ejecutada por un familiar cercano, alcances de la manifestación de voluntad anticipada formalizada en instrumento público y el respeto a la voluntad propia y a la voluntad ajena: autodeterminación terapéutica propia a través del nombramiento de un apoyo (véase tabla 1). A continuación, estos temas se desarrollan con citas de las opiniones vertidas por los entrevistados.

Tabla 1

Resumen de los principales temas y subtemas

\begin{tabular}{|c|c|}
\hline Temas & Subtemas \\
\hline $\begin{array}{l}\text { 1. Respeto de la autonomía de la persona a través } \\
\text { de una declaración de voluntad anticipada } \\
\text { ejecutada por un familiar cercano }\end{array}$ & $\begin{array}{l}\text { 1.1. Respeto a la dignidad personal } \\
\text { 1.2. Salvaguarda de los derechos civiles e intereses } \\
\text { de la persona } \\
\text { 1.3. Necesidad de distinguir la autodeterminación } \\
\text { terapéutica de la eutanasia }\end{array}$ \\
\hline $\begin{array}{l}\text { 2. Alcances de la manifestación de voluntad } \\
\text { anticipada formalizada en instrumento público }\end{array}$ & $\begin{array}{l}\text { 2.1. La capacidad de la persona } \\
\text { 2.2. Opinión favorable hacia las declaraciones de } \\
\text { voluntad anticipada para prever situaciones límites } \\
\text { 2.3. Aplicación para adultos en general }\end{array}$ \\
\hline $\begin{array}{l}\text { 3. Respeto a la voluntad propia y a la voluntad } \\
\text { ajena: autodeterminación terapéutica propia a } \\
\text { través del nombramiento de un apoyo }\end{array}$ & $\begin{array}{l}\text { 3.1. Respeto de la voluntad en base a principios, } \\
\text { valores y creencias personales } \\
\text { 3.2. Facilitar la toma de decisiones en un momento } \\
\text { crítico } \\
\text { 3.3. Evitar conflictos familiares }\end{array}$ \\
\hline
\end{tabular}

Fuente: Elaboración propia a partir de los resultados del estudio.

\section{Respeto a la autonomía de la persona:}

Declaración de voluntad anticipada ejecutada por un familiar cercano:

El primer tema que se identificó en las entrevistas realizadas fue el respeto a la autonomía de la persona a través de una declaración de voluntad anticipada ejecutada por un familiar 
cercano. Los entrevistados tuvieron opiniones coincidentes respecto a la importancia de las declaraciones de voluntad anticipada previamente otorgadas para que sean ejecutadas por un familiar cercano. Por su amplitud, se ha subdividido en tres subtemas: (1) respeto a la dignidad personal, (2) salvaguarda de los derechos civiles e intereses de la persona y (3) la necesidad de distinguir la autodeterminación terapéutica de la eutanasia.

\section{Respeto a la dignidad personal:}

La dignidad de la persona es el punto de partida de esta legislación especializada. Significa un avance para al sistema legal nacional y así lo identifican los entrevistados, resaltando que la misma parte desde el respeto de la dignidad personal, tal como la orienta la propia constitución:

"El artículo 1 de la Constitución Política del Perú dispone que: "La defensa de la persona humana y el respeto de su dignidad son el fin supremo de la sociedad y el Estado." Considero este postulado constitucional como el marco rector e inspiracional de nuestra Carta Magna..."

Asimismo, se destacó la necesidad de garantizar el ejercicio de los derechos civiles como parte del respeto de la dignidad de las personas, para lo cual es el Estado a través de su aparato legislativo, a quien le compete esta labor: "Considero que esta Ley es necesaria para garantizar el ejercicio irrestricto de los derechos y libertades fundamentales de las personas (que la necesiten) en todo momento y cumplan ese propósito aspiracional de la dignidad humana..."

Salvaguarda de los derechos civiles e intereses de la persona:

Si el punto de partida es el respeto de la dignidad de la persona, esta dignidad se ejecuta a través del ejercicio de los derechos civiles. De esta manera, los entrevistados destacaron que la voluntad del sujeto debe prevalecer en todo momento, aun cuando ya no pueda manifestarla de manera plena:

"(...) En esa medida, toda manifestación de voluntad -en el irrestricto ejercicio de los derechos y libertades fundamentales de las personas- debe ser considerada y respetada a plenitud. Por lo que la referida Ley, más allá de precisar enfáticamente la figura de la curatela, garantiza el ejercicio de los derechos civiles de las personas. Incluso cuando ellas ya no estén en la capacidad de hacerlo..."

Necesidad de distinguir la autodeterminación terapéutica de la eutanasia:

A nivel de la población, el concepto de eutanasia está más difundido que el de autodeterminación terapéutica y así lo expresaron los entrevistados. Los mismos llegaron a 
precisar que es necesario legislar adecuadamente los temas referentes a las declaraciones de voluntad anticipada para poder respetar la vida y dignidad humanas y, más aún, para evitar cualquier supuesto relacionado con la eutanasia:

"(...) La autodeterminación terapéutica es muy amplia. Se tendría que precisar si se trata de la autodeterminación terapéutica en fase terminal (cuando se mantiene con vida a una persona de manera artificial) y que ésta tampoco se confunda con la eutanasia. Además, tendría que estar precisada en legislación vigente, de lo contrario un procedimiento de esta naturaleza no sería viable jurídicamente..."

La autodeterminación terapéutica debe ser evaluada en cada caso, tratando siempre de respetar al máximo de la voluntad del declarante, pero cautelando a su vez sus derechos y dignidad:

"Es decir (...), de que exista la posibilidad que esa manifestación de voluntad no sea considerada de manera absoluta (...) se debe respetar bajo determinadas circunstancias, en interés de la persona y de su salud (...) en lo que sea más favorable para ella, que exista esa posibilidad y que no sea tomada dicha declaración de voluntad de manera absoluta y que llegue al absurdo de causar un perjuicio. Y que podría, en todo caso, si llegáramos hasta ese extremo, entrar en discusión un supuesto de eutanasia".

Se entiende que, para dar una determinada DVA, se debe contar con información veraz y pertinente respecto a cada caso en concreto. Esto facilita que se respete la autonomía de la persona y se cumplan los designios señalados de manera anticipada. Igualmente, garantiza que en un determinado momento la dignidad del paciente - como ser humano merecedor de respeto en todo momento pueda ser valorada. Aun cuando su voluntad no pueda manifestarse. Esta voluntad puede ir en todos los sentidos legalmente previstos. Siendo necesario precisar que, para nuestra legislación, la eutanasia sigue siendo un delito y, por lo tanto, imposible de regular mediante una DVA.

Alcances de la manifestación de voluntad anticipada formalizada en instrumento público:

El uso de un instrumento público genera una serie de garantías y efectos a propósito de los instrumentos o documentos de carácter privado. Así, el segundo tema identificado radica en los alcances de la manifestación de la voluntad anticipada formalizada en instrumento público. A su vez, este tema fue desglosado en tres subtemas: (1) la capacidad de la persona, (2) opinión favorable hacia las declaraciones de voluntad anticipada para prever situaciones límites y (3) aplicación para adultos en general. 


\section{La capacidad de la persona:}

La capacidad puede ser entendida como la habilidad que tiene una persona para tomar decisiones sobre diversos aspectos que le son relevantes y que van, desde las facetas más sencillas de la vida cotidiana hasta situaciones verdaderamente complejas. Es precisamente la disminución o la ausencia de ésta la que genera cierta controversia en el momento en que se debe pasar del plano de las suposiciones a las realidades:

"(...) La pregunta que surge es la siguiente: ¿la capacidad tiene que ser necesariamente plena? Desde mi perspectiva, la capacidad puede tener algunas limitaciones. Resultaría importante fortalecer el nombramiento de un curador cuando la persona se encuentra en plenitud de condiciones, de esta manera, en el momento en el que las limitaciones en su capacidad se empiecen a manifestar, este curador ejecute las decisiones que a futuro se tomaron".

Asimismo, se resaltó que no todas las personas están o gozan de la misma capacidad, lo que no ha sido completamente considerado por el legislador:

"(...) Estamos hablando del adulto mayor. ¿y qué pasó con la persona que está en estado de discapacidad? Yo creo que este también es otro rubro (...), porque, así como el adulto mayor, también está la persona con discapacidad, y de alguna u otra forma están emparentados (los argumentos)... Obviamente hay personas que tienen discapacidad, muy limitada, limitada y ligeramente limitada, pero en muchas ocasiones resulta que tiene de una u otra forma la capacidad de prever de que frente a una situación futura es mucho mejor estar representados a través de un curador en quien confíe..."

\section{Opinión favorable hacia las declaraciones de voluntad anticipada:}

\section{Para prever situaciones límite:}

La cercanía de la muerte lleva a las personas a cuestionamientos a todo nivel. Asimismo, es en estas circunstancias en las que se pueden presentar diversos conflictos, no solo respecto a sus relaciones significativas, sino también a nivel interior del sujeto. La declaración de voluntad anticipada puede servir para prever la toma de decisiones en estas situaciones:

"(...) Si tengo todavía la suficiente lucidez e inteligencia para darme cuenta que voy a tener severas dificultades, si el parte médico, si digamos la atención que recibo de peritos profesionales ya me da indicativos categóricos de lo que me puede ocurrir en el futuro, yo creo que para salvaguardar mis derechos e intereses yo acudiría a esta forma jurídica para 
poder preservarlos, porque seguramente no sería yo, sino algunas personas que pudieran estar dependiendo de mí y gracias a la presencia del curador yo podría salvaguardar sus intereses y derechos (...). Esa norma en términos generales es una norma que tutela, que tiene limitaciones, que puede ser ampliada, que puede ser mucho más generosa con respecto a la capacidad de previsión y que está llamada -creo yo-, si es que se le da la difusión correspondiente, a ser una buena salida a los problemas (...)."

No obstante, es importante verificar que siempre los límites de la vida son temas delicados al momento de ser tratados en todos los sentidos culturales:

"Considero que mucho antes de que se tome una decisión final, se debería regular prolijamente el "proceso de la toma de decisión final", por ejemplo, cumplir algunos criterios médicos que garanticen la irreversibilidad de la enfermedad y por ende garantizar que el futuro curador sea finalmente una persona capacitada y preparada para tal fin, con el objetivo de hacer prevalecer la voluntad anticipada de su patrocinado."

La responsabilidad que deriva del acto médico realizado por el personal de salud es un aspecto que también guarda relación con las declaraciones de voluntad anticipada. De estar reguladas de manera positiva, se puede ayudar a direccionar la actuación del personal sanitario:

"El acto médico está en función de ayudar a recuperar la salud, pero en este caso hay situaciones límites donde -como profesional de la salud- le estoy proponiendo, preguntando o considerando cómo debe morir, porque es una declaración anticipada frente a situaciones terminales y ahí, lamentablemente el sistema de salud no tiene una postura muy clara. Así, una declaración de voluntad anticipada, debidamente regulada puede ayudar a humanizar el sistema sanitario, respetando la autonomía y los derechos del paciente."

\section{Aplicación para adultos en general:}

La legislación ha tutelado preferentemente a precisos grupos poblaciones vulnerables, tal vez sin tomar en cuenta que estos temas son de interés general y que podrían ser mucho más necesarios en otros casos que poco tienen que ver con el avance de la edad:

"La norma está dirigida a los adultos mayores, entiéndase a las personas mayores de 60 años. Opino que sería mucho más favorable que la misma sea ampliada, es decir, que se le permita a cualquier persona adulta realizar esta designación -autodeterminarse-, especialmente para las personas que puedan padecer alguna enfermedad degenerativa o progresiva y que afecte su capacidad a futuro, de esta manera podrá tomar decisiones 
relevantes cuando todavía cuenta con la capacidad para hacerlo, y que una persona de su entera confianza (designada por él), sea quien ejecute estas decisiones. Tal como está la norma, una persona en estas circunstancias y con 50 años, por ejemplo, no podría designar un curador".

\section{Respeto a la voluntad propia y a la voluntad ajena:}

Autodeterminación terapéutica propia a través del nombramiento de un apoyo:

Por último, el tercer tema está enfocado hacia el respeto tanto de la voluntad propia como de la ajena: en qué medida se puede respetar la voluntad del sujeto en casos como estos. Se pone de manifiesto la profundidad de las cuestiones espirituales de las personas, donde las decisiones son tomadas siempre a partir de sus valores, creencias y principios. Este tema revela tres subtemas sumamente importantes: (1) respeto a la voluntad en base a principios, valores y creencias personales, (2) facilitar la toma de decisiones en un momento crítico y (3) evitar conflictos familiares.

\section{Respeto a la voluntad en base a principios, valores y creencias personales:}

La cercanía de la muerte de uno mismo o de un familiar cercano es un tema delicado de abordar. Las personas se niegan de manera inconsciente a situarse en dicho escenario, sin embargo, la presencia de una eventual enfermedad que pueda degenerar sus funciones y capacidades las lleva a enfrentarlo. Los entrevistados se presentaron a favor de otorgar una declaración de voluntad anticipada que sea respetada en dicha situación por todo aquel que deba hacerlo. Señalando, además, que esta responde a sus principios, valores y creencias personales:

"Si fuera mi esposa o mis hijos, probablemente el curador sería yo. La declaración de una voluntad anticipada supone un proceso de diálogo, de deliberación, de consultas, para a partir de ahí llegar a una conclusión que no solamente es terapéutica, sino también es una decisión ética, con una dimensión psicológica vivencial. De manera que la persona diga con todo eso, yo quiero esto. Y ciertamente si esto es así, habría de respetarse."

Como se presenta la perspectiva de la pérdida de la capacidad, los entrevistados señalaron la necesidad de la presencia de la familia a manera de soporte en esta circunstancia y para la toma de las decisiones:

"(...) Que un familiar cercano, el más directo asuma aquella responsabilidad. Pero solamente por un hecho especial, de tener una edad muy avanzada y no contar con familiares que puedan velar por mí: sí, sin duda nombraría un curador que no tuviera un vínculo familiar 
conmigo (...). Esto tendría como propósito el respeto de mi voluntad por cuestiones de principios, de valores culturales, de religión, etcétera. Considero que esto debe primar."

\section{Facilitar la toma de decisiones en un momento crítico:}

El beneficio que puede representar una declaración de voluntad anticipada radica no solo en el respeto de la voluntad del otorgante, sino también, a manera de paliativo, en facilitar la toma de decisiones en momentos críticos. Enfocadas desde esta perspectiva las declaraciones de voluntad anticipada se convierten en algo más que un instrumento:

"(...) Porque primero, además de trazar una hoja de ruta en una etapa crítica de mi vida, también evitaría sufrimiento de aquel que tiene que tomar la decisión (...). Imagínese en una situación crítica, no he dejado ninguna declaración de voluntad anticipada, quién toma la decisión también sufre y divide a la familia. (...) Mientras que una declaración de voluntad anticipada puede decir: "yo quiero esto". Puede que estén en contra pero que se respete, ya no cae toda la decisión sobre una persona, que lamentablemente va a tener que cargar con la decisión que a conciencia creyó la más oportuna y porque finalmente alguien tiene que tomar la decisión. Y ese alguien no puede ser, creo yo, absolutamente el médico o el sistema sanitario."

\section{Evitar conflictos familiares:}

La familia es el eje de las relaciones humanas. Dentro del núcleo familiar la toma de decisiones en momentos límite son fuente de constantes desavenencias $y$, muchas veces, puede ocasionar daños en las relaciones que mantienen sus miembros. Sin embargo, se propone que la designación de un curador evitaría en cierta medida la generación de estos conflictos intrafamiliares:

"(...) Sería más conveniente y evitar un proceso judicial, al margen de la voluntad del sujeto, muchas veces los familiares empiezan los procesos a litigar y sobre todo entran en conflicto cuando hay bienes. En ese sentido, primero para tomar precauciones y evitar ese tipo de procesos y conflictos familiares, lo más conveniente es que uno ya más o menos, tomando precauciones a esa situación pueda designar su curador, estoy de acuerdo."

Igualmente, se señaló que pueden garantizar el respeto de la autodeterminación:

"Porque trataría de evitar situaciones límites a mi familia. Entiendo yo que estas son situaciones límites, en la cual la persona se va a ver en una situación cercana al límite de la vida en donde influyen mucho los temas morales, las creencias religiosas, formación. De repente ellos pueden decidir algo que yo no quiero, y no respetar mi voluntad, entonces creo 
que sí debería permitírseme que yo decida a efectos de que en el futuro no les genere alguna complicación a mis familiares."

\section{Discusión}

Considerando los hallazgos generados a partir de las entrevistas realizadas se examinan las perspectivas y opiniones de los profesionales sobre la declaración de voluntad anticipada y sobre cómo esta es una forma de tutela de la autodeterminación que impacta la vida familiar y el accionar del personal sanitario.

Los entrevistados destacan la importancia del respeto a la autonomía de la persona a través de una declaración de voluntad anticipada, la misma que puede o debe ser ejecutada por un familiar cercano previamente designado. En el mismo sentido, un estudio realizado por Howard (2012) concluye que la voluntad de la persona y el respeto de su autonomía deben ser operadas por el "representante" quien ejecuta la voluntad previamente declarada por el otorgante, dado que la legislación de su país -Uruguay-, así lo contempla. De igual manera, Marín (2019), en su tesis de doctorado señala que el representante es la persona designada por el declarante para ser su "interlocutor" y quien debe hacer respetar su autonomía y su dignidad como persona en todo momento.

Conforme se observó, los entrevistados narraron además que este documento puede significar una directriz para el personal de salud, quien ve direccionado su proceder de acuerdo con lo previamente declarado por el otorgante, siempre que este haya perdido la capacidad de poder manifestar su voluntad. La dignidad del paciente quedaría en buena cuenta, respetada por el hecho de aplicar únicamente, aquellos actos médicos que han sido autorizados de manera previa mediante una DVA, siendo el curador, apoyo o representante, quien autorice los formatos de consentimiento informado. La responsabilidad que deriva del acto médico realizado por el personal de salud es un aspecto que también guarda relación con las declaraciones de voluntad anticipada. De estar reguladas de manera positiva, se puede ayudar a direccionar la actuación del personal sanitario (Ciliberti et al., 2018; Scholten et al., 2018). Así también concluye el estudio realizado por el costarricense Marín (2017), en el que se indica que este tipo de documentos, realizados conforme a la legislación del país, brinda beneficios a todo nivel, tanto para el personal sanitario, para el mismo declarante y para la familia que debe atravesar por esa difícil situación.

En Perú, la instrumentalización de esta declaración de voluntad anticipada beneficia exclusivamente a los adultos mayores y a las personas con discapacidad. Los entrevistados coinciden en que la capacidad es relativa y tiene peculiaridades de persona a persona. Es 
decir, que esta responde a diversos factores complejos como el nivel socio cultural y el entorno de la persona, lo que no ha sido oportunamente contemplado por el legislador peruano. Sin embargo, en España, la premisa es que sea la persona con capacidad y plenamente informada quien otorgue estos instrumentos (Bejarano et al., 2019; Jiménez, 2014). Es importante también señalar que en algunos casos el padecimiento de una enfermedad degenerativa es la condición para poder otorgar estas declaraciones (Howard, 2012), hecho que consideramos, no es relevante para poder tomar decisiones a futuro y que, sin embargo, pueden ser determinante en dicha circunstancia. No obstante, el estudio realizado por Bejarano y colaboradores (2019) señaló que, en España, a pesar de existir un conocimiento general sobre las declaraciones de voluntad anticipada y ser considerada en su mayoría como útil, es aún bajo el porcentaje de personas que han llevado a cabo el registro de dicha declaración. Es decir, a pesar de tener conocimientos sobre el tema, pocos los han materializado, siendo necesario que se difundan más estos temas para poder hacer un ejercicio adecuado de los mismos.

Por último, los entrevistados rescatan que las declaraciones de voluntad anticipada son una herramienta valiosa para el respeto tanto de la voluntad propia como de la ajena. Igualmente, se presentaron a favor de otorgar una declaración de voluntad anticipada que sea respetada por todo aquel que deba hacerlo. Señalando, además, que esta responde a sus principios, valores y creencias personales. En un sentido similar, Gómez y Córdova (2016), en un estudio realizado en Colombia, destacan que estos instrumentos, significan un alivio para los familiares, toda vez que los exonera de tomar decisiones en situaciones límite. En otro sentido, también destacado, Marín Mora (2017) expresa que estas declaraciones tienen un gran significado para el otorgante e inclusive para su familia, pues representan una especie de garantía para el respeto de su voluntad y dignidad como personas, minimizando el impacto familiar que tiene esta toma de decisiones sobre la salud de otra persona.

A la hora de generalizar los hallazgos del estudio se debe tener en cuenta entre sus limitaciones que el número de entrevistados fue reducido y que respecto al argumento abordado la entrevista en profundidad debe considerarse como exploratoria (Hernández Sampieri et al., 2004). Por otro lado, si bien se identificaron tres temas centrales, con sus respectivos subtemas a partir de tópicos recurrentes, pudiera no haberse logrado completamente la saturación de los datos (Neuendorf, 2018). Aunque se debe recalcar que el contenido y el enfoque de las entrevistas no dificultaron su interpretación. En este sentido, es una limitación del estudio que entre los modos alternativos para comunicar ideas 
y puntos de vista sobre las declaraciones anticipadas solamente se exploraron a través de la observación, no considerándose, por ejemplo, la historia personal de los entrevistados.

\section{Conclusiones}

La comprensión de la perspectiva de los médicos y abogados constituye un respaldo a la futura discusión sobre las directivas anticipadas colaborando a cerrar las brechas legal y ética en materia que actualmente persisten en la realidad peruana. Las declaraciones de voluntad anticipada pueden ser una herramienta verdaderamente efectiva para asegurar el respeto a la dignidad de la persona, así como una garantía para la observancia de sus derechos civiles. Se ha verificado que las personas consideran más favorable tomar las decisiones sobre su salud de manera anticipada e informada, a que sean terceros quienes decidan por ellos.

En el caso peruano, la legislación ha optado por enfocarse en grupos de población vulnerables, sin embargo, en otras legislaciones, el tratamiento de las declaraciones de voluntad anticipada está generalizado para cualquier sector de la ciudadanía, lo que significa una verdadera garantía en el respeto de la voluntad del sujeto y su autonomía como paciente.

Las declaraciones de voluntad anticipada además pueden servir para direccionar la conducta del personal sanitario, a pesar de que el paciente ya no pueda manifestar su voluntad en el momento requerido, asegurando el respeto a su autonomía previamente declarada. Del mismo modo, constituyen una ventaja para evitar conflictos familiares derivados de la toma de decisiones en los momentos límite de la vida.

Asimismo, son una herramienta importante para que sea respetada la voluntad de las personas, debidamente informadas sobre una situación límite que se podría presentar en el futuro. Ello garantiza, como ya se señaló, el respeto a la dignidad de la persona. Por el contrario, la compromete el ser considerado únicamente como un objeto. Lo que se puede verificar en algunos casos límites cuando las personas no pueden manifestarse a favor o en contra de un determinado acto médico, siendo terceros quienes toman las decisiones. Los cuales, por no caer en responsabilidades y con la finalidad de evitar una demanda o denuncia posterior, se extralimitan en la aplicación de determinados actos médicos.

Tomado en cuenta lo antes expuesto, se recomienda el estudio, la regulación jurídica y la difusión de estas declaraciones de voluntad anticipadas, las mismas que deben ser impulsadas, no sólo por el Estado, sino también a nivel particular, por personal especializado en el estudio de estos temas. La redacción de la declaración debe ser lo suficientemente 
clara como para que no produzca confusión en su ejecución y para que no perjudique al otorgante, garantizando siempre el derecho a su dignidad y autodeterminación.

\section{Referencias}

Bejarano Gómez, M. D. C., Braojos Bautista, R., Díez García, M. R., Prieto Sánchez, M. D. C., \& Sánchez Díez, S. (2019). Declaración de voluntades anticipadas. Un testamento vital para asegurar la autonomía. Gerokomos, 30(3), 119-123. Obtenido de https://scielo.isciii.es/pdf/geroko/v30n3/1134-928X-geroko-30-03-119.pdf

Botti, C., \& Vaccari, A. (2019). End-of-life decision-making and advance care directives in Italy. A report and moral appraisal of recent legal provisions. Bioethics, 33(7), 842-848. DOI: 10.1111 /bioe. 12615

Brighton, L. J., \& Bristowe, K. (2016). Communication in palliative care: talking about the end of life, before the end of life. Postgraduate medical journal, 92(1090), 466-470. DOI: 10.1136/postgradmedj-2015-133368

Brinkman-Stoppelenburg, A., Rietjens, J. A., \& Van der Heide, A. (2014). The effects of advance care planning on end-of-life care: a systematic review. Palliative medicine, 28(8), 1000-1025. DOI: 10.1177/0269216314526272

Ciliberti, R., Gorini, I., Gazzaniga, V., De Stefano, F., \& Gulino, M. (2018). The Italian law on informed consent and advance directives: New rules of conduct for the autonomy of doctors and patients in end-of-life care. Journal of critical care, 48, 178-182. DOI: 10.1016/j.jcrc.2018.08.039

Clarke V., Braun V., Hayfield N. (2015). Thematic analysis. En: Smith J. organizador. Qualitative psychology: A practical guide to research methods. p. 222-48.

Dabove, M. (2018). Autonomy, self-determination, and human rights: legal safeguards in Argentina to prevent elder abuse and neglect. International Journal of Law, Policy and The Family, 32(1), 80-92. DOI: $10.1093 /$ lawfam/ebx017

Decreto legislativo № 1384, 4 de septiembre 2018, Decreto legislativo que reconoce y regula la capacidad jurídica de las personas con discapacidad en igualdad de condiciones. Diario Oficial El Peruano. Obtenido de https://cdn.www.gob.pe/uploads/document/file/192139/DL_1384.pdf

García-Toma, V., \& García-Yzaguirre, J. (2018). Diccionario de derecho constitucional. Lima: Instituto Pacífico S.A.C.

Gómez, L. B., \& Córdoba, I. G. (2016). Voluntades anticipadas al final de la vida. Una aproximación desde la regulación colombiana y en el derecho comparado. Revista Latinoamericana de Bioética, 16(1), 128-153. DOI: 10.18359/rlbi.1444

Hernández Sampieri R., Fernández Collado C., \& Baptista Lucio P. (2004). Metodología de la investigación. México DF: McGraw-Hill Interamericana. 
Heyland, D. K., Heyland, R., Dodek, P., You, J. J., Sinuff, T., Hiebert, T., \& Day, A. G. (2017). Discordance between patients' stated values and treatment preferences for end-of-life care: results of a multicentre survey. BMJ supportive \& palliative care, 7(3), 292-299.DOI: 10.1136/bmjspcare-2015-001056

Howard Zuluaga AM. (2012). Las declaraciones de voluntad anticipada y la autonomía de la persona. Revista de Derecho, 11(21), 171-98. Obtenido de http://revistaderecho.um.edu.uy/wpcontent/uploads/2012/10/DERECHO-21.pdf

Inbadas, H., Carrasco, J. M., \& Clark, D. (2020). Representations of palliative care, euthanasia and assisted dying within advocacy declarations. Mortality, 25(2), 138-150. DOI: 10.1080/13576275.2019.1567484

Inbadas, H., Zaman, S., Whitelaw, S., \& Clark, D. (2017). Declarations on euthanasia and assisted dying. Death studies, 41(9), 574-584. DOI:10.1080/07481187.2017.1317300

Jiménez Muñoz FJ. (2014) Una aproximación a la regulación española del documento de voluntades anticipadas o «testamento vital». Diario La Ley ,22, 5-10. Obtenido de: https://dialnet.unirioja.es/servlet/articulo?codigo=3123059

Kernick, L. A., Hogg, K. J., Millerick, Y., Murtagh, F. E., Djahit, A., \& Johnson, M. (2018). Does advance care planning in addition to usual care reduce hospitalisation for patients with advanced heart failure: A systematic review and narrative synthesis. Palliative medicine, 32(10), 1539-1551. DOI: 10.1177/0269216318801162

Ley $N^{\circ} 28189$, 18 de marzo de 2004, Ley general de donación y trasplante de órganos y/o tejidos humanos. Diario Oficial. Obtenido de https://ww1.essalud.gob.pe/trasplanteweb/pdf/Ley28189.pdf

Ley $\mathrm{N}^{\circ} 29633,18$ de diciembre de 2010, Ley que fortalece la tutela del incapaz o adulto mayor. Diario Oficial El Peruano. Obtenido de https://www.mimp.gob.pe/adultomayor/archivos/Ley29633.pdf

Marín Mora AA. (2017). Las Voluntades Anticipadas: una perspectiva ético-jurídica. Cadernos iberoamericanos de direito sanitário, 6(2), 10-24. DOI: 10.17566/ciads.v6i2.371

Marín Mora AA. (2019). La autonomía del paciente expresada en los documentos de voluntades anticipadas: un análisis desde el bioderecho. Tesis de grado de Magister. Murcia: Universidad de Murcia. Obtenido de http://hdl.handle.net/10201/85124

Monteiro, R. D. S. F., \& Silva Junior, A. G. D. (2019). Advance directive: historical course in Latin America. Revista Bioética, 271), 86-97. DOI: 10.1590/1983-80422019271290

Nehra, D. K., \& Gupta, R. (2019). Advance Directives: identifying possible barriers in implementation and potential remedies. Open Journal of Psychiatry \& Allied Sciences, 10(2), 105-9. DOI: $10.5958 / 2394-2061.2019 .00023 .5$

Neuendorf KA. (2018) Content analysis and thematic analysis. En Brough P. Advanced Research Methods for Applied Psychology. New York: Routledge. p.211-23. 
Nguyen, K. H., Sellars, M., Agar, M., Kurrle, S., Kelly, A., \& Comans, T. (2017). An economic model of advance care planning in Australia: a cost-effective way to respect patient choice. BMC health services research, 171), $1-8 . \quad$ Obtenido de https://bmchealthservres.biomedcentral.com/articles/10.1186/s12913-017-2748-4

Nicholas, L. H., Langa, K. M., Iwashyna, T. J., \& Weir, D. R. (2011). Regional variation in the association between advance directives and end-of-life Medicare expenditures. Jama, 306(13), 1447-1453. DOI: $10.1001 /$ jama.2011.1410

Páramo Bernal P (2013). La investigación en ciencias sociales: estrategias de investigación. Bogotá: Universidad piloto de Colombia.

Rietjens, J. A., Sudore, R. L., Connolly, M., van Delden, J. J., Drickamer, M. A., Droger, M., \& European Association for Palliative Care. (2017). Definition and recommendations for advance care planning: an international consensus supported by the European Association for Palliative Care. The Lancet Oncology, 18(9), e543-e551. DOI: 10.1016/S1470-2045(17)30582-X

Scholten, G., Bourguignon, S., Delanote, A., Vermeulen, B., Van Boxem, G., \& Schoenmakers, B. (2018). Advance directive: does the GP know and address what the patient wants? Advance directive in primary care. BMC medical ethics, 19(1), 1-7. DOI: 10.1186/s12910-018-0305-2

Sophiea, H., \& Davidab, S. (2018). Directing citizens to create advance directives. Swiss Medical Weekly, 148, w14628. DOI: 10.4414/smw.2018.14628

Sudore, R. L., Lum, H. D., You, J. J., Hanson, L. C., Meier, D. E., Pantilat, S. Z., \& Heyland, D. K. (2017). Defining advance care planning for adults: a consensus definition from a multidisciplinary Delphi panel. Journal of pain and symptom management, 53(5), 821-832. DOI: $10.1016 / j$.jpainsymman.2016.12.331

Torres Morales S. (2015) El testamento vital: una genuina manifestación de la autonomía de la voluntad. Lumen, (11), 59-75. DOI: 10.33539/lumen.2015.n11.545

Tulsky, J. A., Beach, M. C., Butow, P. N., Hickman, S. E., Mack, J. W., Morrison, R. S., ... \& Pollak, K. I. (2017). A research agenda for communication between health care professionals and patients living with serious illness. JAMA internal medicine, 1779), 1361-1366. DOI: 10.1001/jamainternmed.2017.2005

Vaismoradi M., Jones J., Turunen H., Snelgrove S. (2016). Theme development in qualitative content analysis and thematic analysis. Journal of Nursing Education and Practice, (5), 100-110. DOI: 10.5430/jnep.v6n5p100

Varsi-Rospigliosi E. (2001). Derecho médico peruano. Lima: Universidad de Lima. Fondo de Desarrollo editorial. 


\section{Cómo citar este trabajo}

Enriquez Canto, Y., \& Katia Denisse, C. G. (2021). Perspectivas de profesionales peruanos sobre las declaraciones de voluntad anticipada. Apuntes De Bioética, 4(1), 102-121.

https://doi.org/10.35383/apuntes.v4i1.584

\section{Financiación}

El presente artículo no cuenta con financiación específica para su desarrollo y/o publicación.

\section{Conflicto de interés}

Los autores del artículo declaran no tener ningún conflicto de intereses en su realización.

(c) Los autores. Este artículo es publicado por la Revista Apuntes de Bioética del Instituto de Bioética, Universidad Católica Santo Toribio de Mogrovejo. Commons Atribución-NoComercial-CompartirIgual 4.0 Internacional (CC BY-NC-SA 4.0), que permite el uso no comercial, distribución y reproducción en cualquier medio, siempre que la obra original sea debidamente citada. 